\title{
Characterization of the discharge Coefficient Of a sonic VENTURI NOZZLE
}

\author{
A. Cruz-Maya', F. Sánchez-Silva². P. Quinto-Dlez² \& M. Toledo-Velázquez² \\ 1 Programa Integral de Investigación en Gas. Instituto Mexicano del Petróleo, \\ Eje Central Lázaro Cárdenas 152, Col. San Bartolo Atepehuacan, 07730 Mexico DF., \\ Mexico. Unidad Profesional Interdisciplinaria de Ingeniería y Tecnologias Avanzadas, \\ Departamento de Mecatrónica, IPN. \\ ${ }^{2}$ Laboratorio de Ingenieria Térmica e Hidráulica Aplicada, Sección de Estudios de Posgrado e Investigación, \\ Escuela Superior de Mecánica e Ingeniería Electrónica, Instituto Politécnico Nacional. \\ Av. IPN s/n Col. Lindavista, Edif. 5, UPALM, 07738, Mexico, DF., Mexico. \\ jcmaya@imp.mx
}

Received: November $17^{\text {st }}, 2003$. Accepted: May $24^{\text {th }}, 2005$

\section{ABSTRACT}

This paper identifies and determines the main parameters used to calculate the discharge coefficient of a toroidal Venturi nozzle according to the ISO Standard 9300, operating at the critical flow regime (sonic). This study was conducted to investigate the effects of the viscous stresses in the turbulent boundary layer, the wall thermal boundary condition, and the flow field curvature at the nucleus of the nozzle on the discharge coefficient by means of a theoretical and numerical approach. Characterization of the discharge coefficient in the Venturi sonic nozzle was performed considering the viscous and multidimensional effects of the fluid flow as uncoupled phenomenon. As a result, each non-ideal mechanism can be analyzed independently from the influence of the other mechanism. We present a numerical procedure to characterize the discharge coefficient in the inviscid region of the flow, by using the numerical simulation of the inviscid main flow by means of the commercial CFD code. In the region of the viscous stresses, the characterization of this coefficient is based on the analytical theory of the turbulent boundary layer. This characterization allowed obtaining a correlation of the discharge coefficient that was validated by direct comparison between the experimental correlations of the discharge coefficient in turbulent boundary layer proposed by ISO-9300 and the Korea Research Institute of Standards and Science (KRISS). This validation was carried out for throat Reynolds numbers from 1.4 to $2.6 \times 10^{6}$. The agreement of the theoretical and measured discharge coefficients by these correlations was better than $0.2 \%$.

\section{RESUMEN}

En el presente artículo se identifican y caracterizan los parámetros que intervienen en la determinación del coeficiente de descarga de toberas toroidales de flujo crítico bajo la norma ISO-9300. El estudio se enfoca a investigar la incidencia de los efectos viscosos en la región de capa límite y la curvatura del campo de flujo en el núcleo de la tobera sobre el coeficiente de descarga. La caracterización del coeficiente de descarga se lleva a cabo dividiendo el flujo en dos zonas para su estudio, la primera zona considera los esfuerzos viscosos generados en la región de capa límite, en la segunda zona, el flujo se considera no viscoso y el estudio se orienta hacia su comportamiento no unidimensional. Cada uno de estos mecanismos es estudiado en forma independiente sin la influencia del otro, la caracterización del coeficiente en la región de flujo no viscoso se lleva a cabo mediante la simulación numérica del flujo por medio de CFD. En la región de flujo viscoso, la caracterización del coeficiente se lleva a cabo bajo un enfoque analítico, basado en la teoría de capa límite turbulenta. Como resultado de la caracterización, se obtiene una correlación teórico-numérica capaz de determinar el coeficiente de descarga directamente sin necesidad de calibración. La correlación obtenida fue validada mediante una comparación directa contra correlaciones experimentales obtenidas en el Korea Research Institute of Standards and Science (KRISS) y la 
norma ISO-9300 para números de Reynolds en la garganta de la tobera de 1.4 a $2.6 \times 10^{\circ}$, la desviación es inferior al $0.2 \%$.

KEYWORDS: Critical Nozzle, Discharge Coefficient, Critical Flow.

\section{INTRODUCTION}

A Venturi sonic nozzle is a well-accepted and utilized flow measurement device in most high-pressure gas test facilities. It is accurate and its measurement uncertainty is well documented in the ISO 9300-1990 standard [1]. These devices have numerous industrial applications, such as mass flow controllers, and pressure isolators, besides being used as calibration standards for other gas flow meters. The development of the critical flow nozzle has now reached the mature stage, where it can be used as a reference flowmeter and to give traceability to the National Standard of Flow Measurement. Representative critical nozzle geometry, as recommended by the ISO 9300 Standard, was selected for the base line of this study. Figure 1 shows the nozzle shape.

$4 d$

A

$2 \mathrm{~d}$

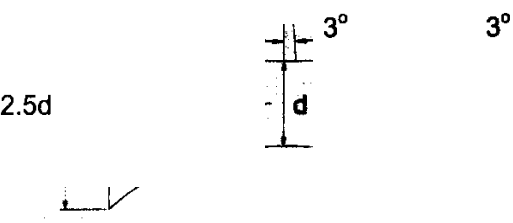

Figure 1. Geometny of the critical nozzle investigated

The main advantage of the nozzle is its extremely simple construction, without any moving parts, that may produce friction or wear, leading to its long-term stability and excellent repeatability. Another important advantage is that the critical flow prevents the propagation of pressure disturbances downstream of the throat nozzle. However, the accuracy of the nozzle depends largely on whether or not the determination of its discharge coefficient is accurate; the discharge coefficient plays an important role in gas flow measurement by means of critical venturi nozzles. It is known that the discharge coefficient of the Venturi nozzle is defined as the ratio of the actual flowrate to onedimensional ideal flowrate. This coefficient should be characterized for viscous boundary layer and flow field curvature (basically, two dimensional) effects. Therefore, it is always less than one, although quite close to the unit.

When a compressible fluid flows through a converging then diverging channel (shown in figure 1), velocity reaches a maximal value at the point of minimal flow area (throat sonic nozzle). The throat velocity increases as the overall differential pressure across channel increases. The limiting value of throat velocity is the speed of sound; further increases in overall differential pressure will not result in an increase in throat velocity. When the throat velocity reaches the speed of sound this is called critical flow $(M=1)$ and the nozzle is said to be choked. The presence of critical flow can be confirmed by monitoring the critical pressure ratio. The critical flow Venturi is a converging then diverging channel configured as a flow measurement device. When the flow is critical, the volumetric flowrate in the throat is a function of the speed of sound, the throat area, and the calibration constant. The mass flowrate is a function of the volumetric flowrate and the fluid density in the throat. Although increasing the stagnation inlet pressure, $P_{o}$, will not affect the volumetric flowrate, the mass flowrate will increase in proportion to the fluid density. With appropriate pressure and temperature measurements, a sonic nozzle can be used to measure mass flowrate. The mass flowrate will remain constant, if the stagnation pressure and temperature and composition remain unchanged. 


$$
m_{\text {iddeal }}=\frac{P_{o} A^{*} C^{*}}{\sqrt{R T_{o}}}
$$

The analytical model is only valid for critical flow $(M=1)$, the mass flowrate depends on measurement of the inlet pressure $P_{o}$ and the temperature $T_{o}$, their values must be expressed in absolute units. The variables $A$ and $R$ are the throat area and the ideal gas constant. The critical flow factor $C^{*}$ represents the thermodynamic change in the isentropic flow from the stagnation condition to the sonic condition and it depends of the thermo-physical properties of the gas at the throat sonic nozzle, and the upstream temperature and pressure. The work of Johnson [2] represented a breakthrough in accurate computation of $C^{*}$ for real gases. The ideal mass flowrate model given by equation (1) provides a convenient first order estimate of the actual mass flowrate through a critical sonic nozzle (I.e., $1-10 \%$ of reading, depending on Reynolds number). A better agreement between theory and experiments can be obtained by including into the ideal model, various non-ideal mechanisms that affect the actual mass flowrate. These non-ideal mechanisms include the viscous effects in the boundary layer and the multi-dimensional behavior of the flow.

To obtain a better accuracy in the mass flowrate measurement provided for an ideal model, expressed in equation 1. the discharge coefficient of the nozzle $C_{d}$ must be included in this equation. This parameter plays an important role on flow metrology using sonic nozzles; $C_{d}$ attempts to account for deviations due to effects of multidimensional flow (viscous effects on the boundary layer reglon and the effect of a curved sonic surface). The discharge coefficient compensates those effects that strike the ideal behavior of the flow.

The discharge coefficient of the sonic nozzle is defined as the ratio of the actual mass flowrate to the theoretical flowrate corresponding to ideal one-dimensional and isentropic flow, as shown below by equation 2

$$
C_{d} \equiv \frac{m_{\text {actual }}}{m_{\text {ideal }}}
$$

In this equation, the actual mass flowrate is defined by the real mass flowrate that passes through the sonic nozzle; equation (2) shows that $C_{\boldsymbol{d}}$ is a calibration factor of the nozzle. For a given nozzle geometry, the discharge coefficient changes as a function of the flowrate passing thought it.

$C_{d}$ is usually expressed as a function of the Reynolds number based on the throat diameter and the stagnation viscosity of the fluid $\mu_{0}$. as follows

$$
\operatorname{Re}_{d}=\frac{4 m_{\text {actual }}}{\pi d \mu_{o}}
$$

The value of $C_{d}$ is determined from calibrating the sonic nozzle by comparison with a primary flow standard. The uncertainty in $C_{d}$ is, therefore, the same as the uncertainty in the calibration process. An equation for the calibration process can be written based on equation 1, as shown below

$$
C_{d}=\frac{m_{\text {actual }} \sqrt{R T_{o}}}{P_{o} A^{*} C^{*}}
$$

where $\boldsymbol{m}_{\text {actual }}$ is the actual mass flowrate based on the primary flow standard. 
The uncertainty in $C_{d}$ from equation (4) depends on the uncertainties in four components: $m_{a c t u a l}, P_{o}, T_{o}$, and $C^{*}$ and is not a function of $A^{*}, d$, or $R$, because these values are kept constant whenever the sonic nozzle is used or calibrated.

Nowadays, this coefficient is determined experimentally through the primary flow standard system; the experimental calibration process is very expensive, because of the big infrastructure needed to carry it out. In order to eliminate the calibration procedure, some researchers have developed analytical and numerical models to determine the discharge coefficient under different operational conditions. This coefficient is presented as a function of fluid parameters, nozzle geometry, and the Reynolds number based on the throat nozzle diameter. However, these models have focused on the laminar boundary layer flow [3- 6]. The characterization of the discharge coefficlent, carried out in this work, allows obtaining a new correlation of this coefficient, providing a reliable calibration method at a low cost and applicable to any established operational condition. It is important to note that the experimental discharge coefficients are obtained under certain conditions established during the calibration process that can be different of the operational ones. Then, the discharge coefficient could change.

\section{THEORETICAL BASIS}

The characterization of the discharge coefficient is dependent on the shape of the Venturi sonic nozzle and the Reynolds number based on its throat diameter. It appears convenient to express one equation of the discharge coefficient in form of defect. i.e., the total defect in the discharge coefficient consists of two parts: one is caused by radially non-uniform (two dimensional) velocity distribution of the inviscid main flow and the other is due to the viscous boundary layer effect. The corrections for non-idealities due to multidimensional effects and viscous effects of the gas are considered as higher order terms that are subtracted from the baseline (unity). According to the aforementioned. this consideration can be written as

$$
C_{d}=1-\Delta C_{d-i n v}-\Delta C_{d-v i s}
$$

where $\Delta C_{d-i n v}$ is the $C_{d}$ decrement due to the multidimensional effects of the flow nucleus, $\Delta C_{d-v i s}$ is the $C_{d}$ reduction due to the flow viscous effects in the boundary layer. This expression considers the viscous and multidimensional effects of the fluid gas as uncoupled phenomena. The combined effect of these non-ideal mechanisms is modeled as a superposition of the individual effects. As a result, each of the non-ideal mechanism can be analyzed independently from the influence of the other mechanism.

Mathematically, the discharge coefficient can be expressed as

$$
C_{d}=1-\left(1-C_{d-i n v}\right)-\left(1-C_{d-v i s}\right)
$$

This equation does not represent a real gas behavior, due to the compensation of critical flow factor $C^{*}$, since this behavior is compensated in the critical flow factor. The analytical characterization of the discharge coefficient consists simply in determining the non-ideal effects at the throat sonic nozzle.

The discharge coefficient can be calculated for an uncoupled form when the order of magnitude of the multidimensional effects is small and the boundary layer thickness at the throat of the nozzle is also small as compared to the nozzle radius. For a nozzle curvature radius of at least twice the throat diameter these effects are minimal $[7]$.

According with equation (6), the flow can be divided in two regions for the discharge coefficient study. 
VIscous Stress Reglon: In this zone, the discharge coefficient depends on boundary layer growth and viscous stress. The discharge coefficient in the viscous reglon can be determined starting from the displacement thickness analysis of the boundary layer $\delta^{*}$. Under this approach, calculus of the discharge coefficient in this zone depends on the following factors: Specific heat ratio $\gamma$. Prandtl number $P_{r}$, standard radial temperature distribution at the throat nozzle $T_{w} / T^{*}$, compressible factor form $H_{c}$, momentum thickness of the boundary layer $\theta$, displacement thickness of the boundary layer $\delta$, Reynolds number of the throat sonic nozzle, gas constant $R$, and nozzle geometry, as shown in the following correlation:

$$
C_{d-v i s}=f\left(\gamma, P_{r}, T_{w} / T_{o}, \operatorname{Re}_{d}, H_{c}, H_{i}, \theta, \delta^{*}, R, \text { geometry }\right)
$$

Inviscld Flow Reglon: In this region, the flow is irrotational, therefore the discharge coefficient is a function of the distortion of the profile velocities in the nucleus of the flow, and the discharge coefficient only depends on the specific geometry of the nozzle and the specific heat ratio $\gamma$ of the flow, as shown in the following expression:

$$
C_{d-i n v}=f(\gamma, \text { geometry })
$$

These factors are used to determine the discharge coefficlent for gases that behave nearly ideally over the range of thermodynamic conditions typical of critical nozzle flows. However, the above expression can also be used where real gas effects are important by using the critical factor flow $C^{*}$ determined by Johnson [2].

Another phenomenon strikes on the discharge coefficient during flow acceleration from the stagnation point to sonic conditions. The phenomenon is characterized by a reversible thermal energy transformation, so temperature decreases while gas is accelerated through the convergent section of the nozzle. In small-scale nozzles, flow acceleration takes place in very small distances; consequently the flow residence time in the nozzle is not sufficient to reach equilibrium between the vibratory energy of fluid molecules and the thermodynamic environmental conditions. This phenomenon is known as vibratory relaxation, it affects considerably polyatomic gases $(\gamma=1.33)[7]$.

\section{DISCHARGE COEFFICIENT DEFINITION}

\section{Discharge coefficient in turbulent boundary layer}

The presence of viscous effects in the nozzle tends to decrease the mass flowrate below its ideal value given in equation (1). Physically, the no-slip condition existing at the nozzle wall results in a layer of slow moving fluid adjacent to the rigid wall (i. e., the boundary layer). Furthermore, within the boundary laver the fluld's kinetic energy is irreversibly converted into internal energy (I. e., viscous dissipation) so that the boundary layer temperature is higher than the free stream temperature. The higher temperature throughout the boundary layer results in a decrement of fluid density near the rigid wall. Together, the lower than ideal values of fluid velocity and density in the boundary layer yleld a reduction in mass flowrate from that predicted by the one-dimensional inviscid flow solution. The viscous effects can be accounted for by defining an effective throat area in such a manner that the mass flow predicted by one-dimensional inviscid flow theory is equivalent to the actual value of mass flow through the nozzle.

The calculus of the discharge coefficient in the boundary laver zone begins with the following premise:

The mass flowrate that would pass through a nozzle in absence of viscous stresses is bigger than the one it would pass when boundary layer exists"

Considering the previous statement, the mass flowrate decreases due to the viscous stresses and it can be evaluated by subtracting the area where viscous stresses exist from the total throat area. Such a procedure takes away, the boundarv laver displacement thickness $\left(\delta^{*}\right)$. This parameter is defined as the distance that the rigid wall would have to 
move in a flow in order to generate the mass flowrate reduction due to the boundary layer. In fact, it takes into account an effective area. The displacement thickness $\left(\delta^{*}\right)$ can be expressed as follows

$$
\delta^{*}=\int_{n}^{\infty}\left(1-\frac{u}{U}\right) d y
$$

In equation (7), the term $(u / U)$ represents the diminution of the velocity flow in the throat sonic nozzle due to the presence of the boundary layer, where $u$ is the flow velocity in the boundary layer and $U$ is the flow velocity outside the boundary layer. Mathematically, the effective throat radius equals the actual throat radius minus the displacement thickness $\left(\delta^{\prime}\right)$. One-dimensional isentropic flow theory can be used to determine the viscous mass flowrate once the nozzle's rigid wall is displaced inward by $\left(\delta^{*}\right)$. Given that the density and flow at the sonic throat are independent from the throat size, the viscous discharge coefficient can be defined as the ratio of the effective nozzle throat area to the actual nozzle throat. In this way, the discharge coefficient in the region of viscous flow can be related with the boundary layer displacement thickness $\left(\delta^{*}\right)[8]$, as expressed in equation (8)

$$
C_{d-v i s}=1-\frac{4 \delta^{*}}{d}
$$

In the equation (8), the discharge coefficient is a function of the viscous stresses only, under a one-dimensional focus, $d$ is the throat sonic nozzle diameter. The viscous discharge coefficient equation (8) it can be replaced in the equation (6). in order to obtain the viscous and inviscid discharge coefficient, as follows

$$
C_{d}=C_{d-i n v}-\frac{4 \delta^{*}}{d}
$$

In the previous equation, $C_{d-i m v}$ is undefined. The displacement thickness has been determined previously by means of the turbulent boundary layer theory under a two-dimensional focus, for gases with $\operatorname{Pr}=0.7, \gamma=1.4$, and critical conditions, these characteristics are typical of most of the gases quantified using sonic nozzles. The displacement thickness determination is given in more detail in reference [8]. Substitution of the displacement thickness in equation (9) characterizes the viscous discharge coefficient, as follows

$$
C_{d}=C_{d-i n \nu}-0.09970 \operatorname{Re}_{d}^{-0.2113564}
$$

\subsection{Discharge coefficient in inviscid flow}

In this section, the discharge coefficient in the nucleus of the sonic nozzle is determined. In this zone, the flow is free from viscous stresses; therefore, the discharge coefficient depends only on the curvature of the flow in the nozzle and the type of fluid. The ideal flow model approximates the nozzle flow as a one-dimensional flow field; actually, the nozzle flow is multi-dimensional. These multi-dimensional effects result in a curvature of the sonic line in the radial plane of the throat sonic nozzle, this curvature reduces the actual mass flow relative to $m_{\text {ideal }}$. In multi-dimensional flows (neglecting viscous effects), the sonic line follows an approximately parabolic profile that begins just upstream of the throat along the nozzle wall and extends downstream into the diverging section of the nozzle to its vertex on the centerline. Consequently, the flow at the throat cross-section will have, in general, a supersonic velocity near the wall (i. e., $M>1$ ), and a subsonic velocity near the centerline (i. e., $M<1$ ). On the other hand, the sonic line is flat for one-dimensional inviscid flow so that a uniform sonic velocity (i. e., $M=1$ ) exists everywhere along the throat crosssection. This effect can be observed by means of the numerical simulation of the flow in the sonic nozzle by means of the CFD, as shown in figure 2 . 


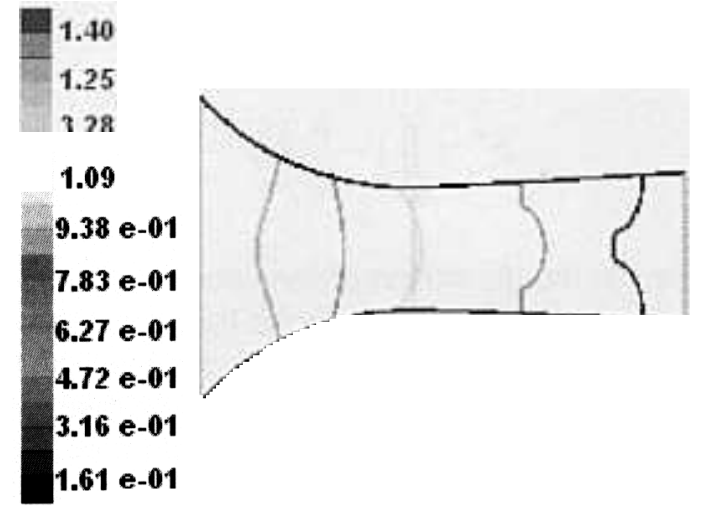

Figure 2.Computational simulation of the iso-Mach lines in sonic nozzle

Based on isentropic flow theory, the mass flux gets a maximal value at a Mach number of unity. Therefore, the mass flowrate is greater for one-dimensional flow where the critical flow condition exists uniformly across the radial plane of the throat sonic nozzle. The viscous effects are not significant outside the boundary layer so the flow can be considered as ideal or isentropic at the core flow. In this way the discharge coefficient is an exclusive function of multidimensional flow behavior due to the centrifugal forces generated by the nozzle reduction. There is a pressure gradient at the throat that yields an opposite force with the same magnitude as the centrifugal force acting on a control volume flow in order to maintain stationary conditions. Hence, the discharge coefficient depends only on the non-uniform radial distribution of field flow because the nozzle is a symmetric element. The field flow distortion can be evaluated by numerical simulation using a 2-D dimensional approach.

The calculus of discharge coefficient begins applying Newton's second law to a fluid element in a normal direction to the streamline, in the throat zone. The procedure ends obtaining the Euler's equation, which represents the radial pressure for a non-viscous flow at the throat of the nozzle, as shown by equation (11)

$$
\frac{\partial p}{\partial Y}=-\frac{\rho v^{2}}{r}
$$

where $Y$ is the radial distance from the nozzle axis to any point of the flow, $p$ is the pressure, while $r$ is the curvature radius respect to some reference point outside the nozzle.

Equation (11) can be integrated through the dimensionless radial distance $Y, Y=Y / Y_{w} ;\left(Y_{w}=d / 2\right)$ between axes and the wall of the nozzle, in the radial plane of the throat sonic nozzle, for a nozzle with constant curvature radius $(r=2 d)$, as shown in figure 3 and equation (12)

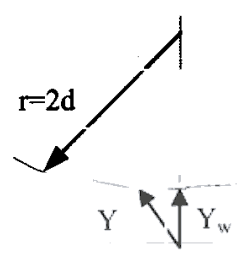

Figure 3. Geometric parameters of the sonic nozzle 


$$
\left[\frac{1}{\bar{Y}_{2}^{2}}-\frac{1}{\bar{Y}_{1}^{2}}\right] B=-\left(\frac{\boldsymbol{v}^{2}}{\boldsymbol{p}_{\mathbf{2}}-\boldsymbol{p}_{1}{ }_{\boldsymbol{M}=1}}\right.
$$

This correlation indicates that the term $\rho v^{2} / p$ depends only on the Aozzle curvature. This term changes according to the radial position of the flow through the symmetric line of the nozzle for a constant curvature radius. Thus, the discharge coefficient for multidimensional flow can be determined as a function of the change of the thermodynamic properties $(\rho, v \mathrm{y} p)$ during the course of the flow through the convergent section of the nozzle until it arrives to the critical condition (radial plane at the throat nozzle). Factor $B$ is a geometric constant of the nozzle; for a curvature radius equal to $2 d, B$ takes a value of 8 .

In the nucleus of the flow neither viscous dissipation nor friction exists; therefore, the flow is considered isentropic and Ideal. The discharge coefficient in this zone can be obtained by means of numerical simulation of an ideal and isentropic flow, under this condition the viscosity is worthless and the Reynolds number grows to infinite value $(\operatorname{Re} \rightarrow \infty)$. Under this consideration, the stagnation conditions of the flow remain unalterable through the nozzle and this is very useful to define a reference state of the fluid. Stagnation conditions can be considered as a reference state because it is independent from the fluid properties at the flow process. Theoretically, for each point along the flow, there is a stagnation state which is defined by hypothetical flow deceleration. So, stagnation conditions are a local function that can be determined easily at each point. Considering an ideal flow starting from the stagnation point, the term $\rho v^{2} / p$ is zero at the entrance of the nozzle. This ratio increases its value while the flow crosses the convergence section of the nozzle and takes a maximal value at the throat when critical flow conditions are attained $(M=1)$. In the throat sonic nozzle, the speed of the flow is similar to the speed of the sound in the flow, as shown in the next relationship: $\rho v^{2} / p=\rho a^{2} / p$. Considering an ideal one-dimensional flow, the discharge coefficient on the core of the flow can be expressed in function of the term $\rho a^{2} / p$, this parameter is evaluated under the stagnation condition of the fluid in the radial plane of the throat sonic nozzle. In this context, the discharge coefficient will take the value of 1 ; the following expression shows the analytical conception of the discharge coefficient

$$
\left.C_{d-i n v}=\frac{\rho_{o}}{\rho^{*}} \frac{a_{o}^{2}}{a^{* 2}} \frac{p^{*}}{p_{o}}\right]_{t}=1
$$

On the other hand, the term $\rho a^{2} / p$ is similar to the ratio of the specific heats $(\gamma)$, considering ideal and onedimensional flow. This takes place only in the radial plane of the throat sonic nozzle, when the speed of the fluid is equal to the speed of the sound. Under this consideration, equation (13) can be expressed as a function of the specific heat ratio $(\gamma)$, as shown in the following equation

$$
\left.C_{d-i n v}=\mid \frac{\rho_{o}}{\rho^{*}} \frac{a_{n}^{2}}{a^{* 2}} \frac{p}{p_{o}}\right]_{t}=\left(\frac{\gamma_{o}}{\gamma}\right)=1
$$

This expression shows that the discharge coefficient in the core of the flow can be calculated taking as reference the relationship of specific heats of the fluid $(\gamma)$. Considering ideal flow, the term $\left(\rho_{o} a_{o}^{2} / p_{o}\right)_{t}$ will remain unalterable on the field flow; this situation takes this parameter as an excellent reference to determine the discharge coefficient in the core flow, under two-dimensional and three-dimensional focuses. The deviation undergone by the value of the discharge coefficient can be determined starting from the two-dimensional focus of the term $\rho^{*} a^{* 2} / p^{*}$; this term refers to the conditions of stagnation of the fluid as shown in the following expression 


$$
C_{d-i n v}=\left(\frac{\gamma_{o}}{\gamma^{*}} \frac{\rho_{2 D}}{\gamma_{o}} \frac{a_{2 D}^{2}}{p_{2 D}}\right)_{t}
$$

where sub index $2 D$ represents the thermodynamic properties of the two-dimensional nozzle flow at the radial plane of the throat sonic nozzle. The terms $\left(\gamma_{o}\right.$ and $\left.\gamma^{*}\right)$ remain unalterable in the radial plane of the throat; however, the term $\left(\rho_{2 D} a_{2 D}^{2} / p_{2 D}\right)$ t takes different values along this plane. For its determination it is necessary to carry out a numerical integration on the radial plane, as shown by the following expression

$$
C_{d-i n v}=\int_{0}^{1}\left(\frac{\gamma_{o}}{\gamma^{*}} \frac{\rho_{2 D}}{\gamma_{o}} \frac{a_{2 D}^{2}}{p_{2 D}}\right)_{t} d \bar{Y},
$$

where $\gamma_{o}$ is the specific ratio reference at the stagnation conditions in the throat, $\gamma^{*}$ is the specific heat ratio at the throat under ideal and critical conditions, $Y$ is the dimensionless radial distance (with respect to the diameter " $d$ " of the sonic nozzle) and sub-index " $t$ " indicates the radial plane of the throat sonic nozzle.

\section{Numerical approach}

In this section, the determination of the discharge coefficient in the nucleus of the nozzle is carried out; the inviscid discharge coefficient depends only on the curvature of the flow in the nozzle and the type of fluid.

To carry out the numerical integration of equation (16) in the radial plane of the throat sonic nozzle, it is necessary to carry out the numerical simulation of the profiles of density, pressure, and velocity of the sound in the nucleus of the sonic nozzle. In this zone, the flow is free of the viscous stresses (inviscid flow). This study was conducted using the commercial CFD code; the numerical solution of the compressible Navier-Stokes equations is obtained by means of implicit time marching procedure. In this approach, the time derivatives are retained in the equations of motion and used to march the solution to a steady state. As a first step of the numerical solution, the Navier-Stokes equations are transformed to a generalized body-fitted coordinate system. The governing equations to be solved in the present simulations are the two-dimensional or axisymmetric compressible Navier-Stokes equations. They are, in a simplified vector form in general, body-fitted coordinates $\xi$ and $\eta$ in the weak conservation law form, as follows

$$
\frac{\partial Q}{\partial \tau}+\frac{\partial F}{\partial \xi}+\frac{\partial G}{\sim}+\alpha H=0
$$

In this expression, the independent variables in physical space $(x, y, t)$ were transformed to a new set of independent variables in computational space $(\xi, \eta, \tau), Q$ is the heat flux, $F, G$ and $H$ are the flux vectors and $\alpha$ represents twodimensional axisymmetric flow. The above. equations are given in more detail by Steger [9]. These generalized equations then are integrated in space and time; the resulting implicit system is solved by alternating directions using implicit factorization. The equation system is closed by using the ideal gas equation of state to relate the fluid density to the pressure and temperature.

\subsection{Computational domain and boundary conditions}

The contour of the axisymmetric converging-diverging nozzle has a converging section consisting of a circular arc that passes through the throat to a point of tangency at which the shape becomes conical. The grid for this geometry consisted of 222 grid points in the axial direction and 1010 grid points in the radial direction. The grid cells in the radial direction were concentrated near the wall in order to resolve the high gradients of thermodynamic properties present in the boundary layer region. For this study the upstream piping section was excluded so that the computational domain included only the converging-diverging nozzle region. Previous investigations show that including the inlet pipe section has a small effect on $C_{d}$ by approximately $0.1 \%$ and can be neglected [7]. For this reason, the piping 
sections are not considered in the numerical study. Boundary conditions are specified at the nozzle inlet and outlet, along the nozzle wall and the symmetric axis. At the nozzle inlet the conditions of stagnation are specified $\left(P_{o}\right.$ y $\left.T_{o}\right)$, at the nozzle outlet the pressure and Mach number are also specified. Symmetric conditions are applied along the centerline; in the nozzle wall the adiabatic and the no-slip boundary conditions are given. Figure 4 shows the discretized mesh of the computational domain of the nozzle by means of the grid generator GAMBIT ${ }^{\circledR}$.

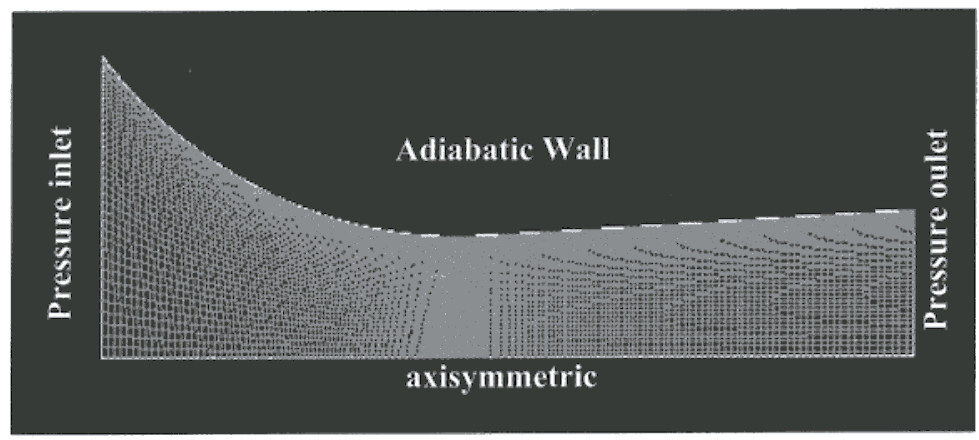

Figure 4. Computational grid for the critical nozzle

The profiles of density, pressure, and velocity of the flow are obtained by means of the numerical simulation of the flow using the commercial numerical code CFD; these profiles are shown in figures 5, 6, and 7.

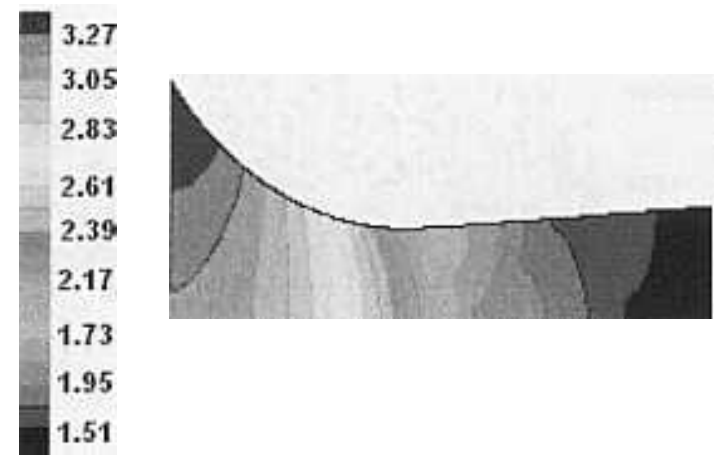

Fiqure 5. Computational results for the 2-D nozzle. Contours of density $\left(P_{o}=2\right.$ bar and $\left.T_{o}=293.15 \mathrm{~K}\right)$

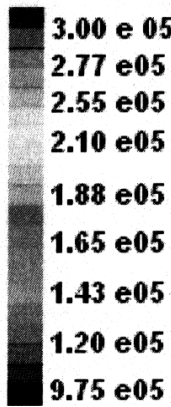

Figure 6. Computational results for the 2-D nozzle. Contours of pressure $\left(P_{0}=2\right.$ bar and $\left.T_{0}=293.15 \mathrm{~K}\right)$ 


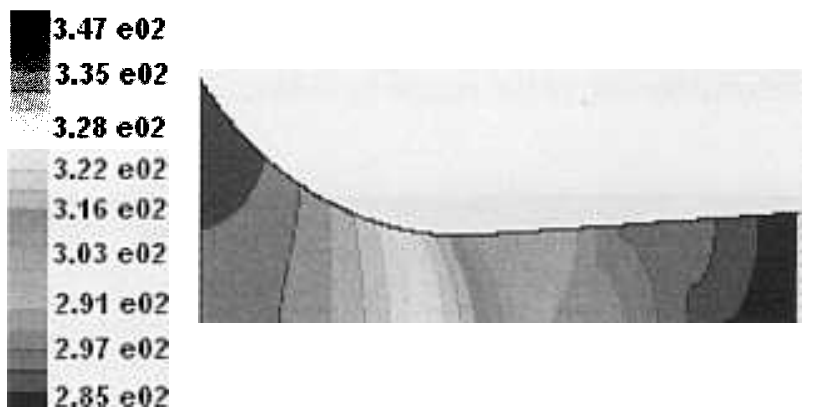

Figure 7. Computational results for the 2-D nozzle. Contours of speed of the sound ( $P_{o}=2$ bar and $T_{o}=293.15 \mathrm{~K}$

In order to determine the inviscid discharge coefficient expressed in equation (16), it is necessary to obtain the term $\left(\rho_{2 D} a_{2 D}^{2} / p_{2 D}\right)_{t}$ in the radial plane of the throat sonic nozzle. This term is shown in figure 8 .

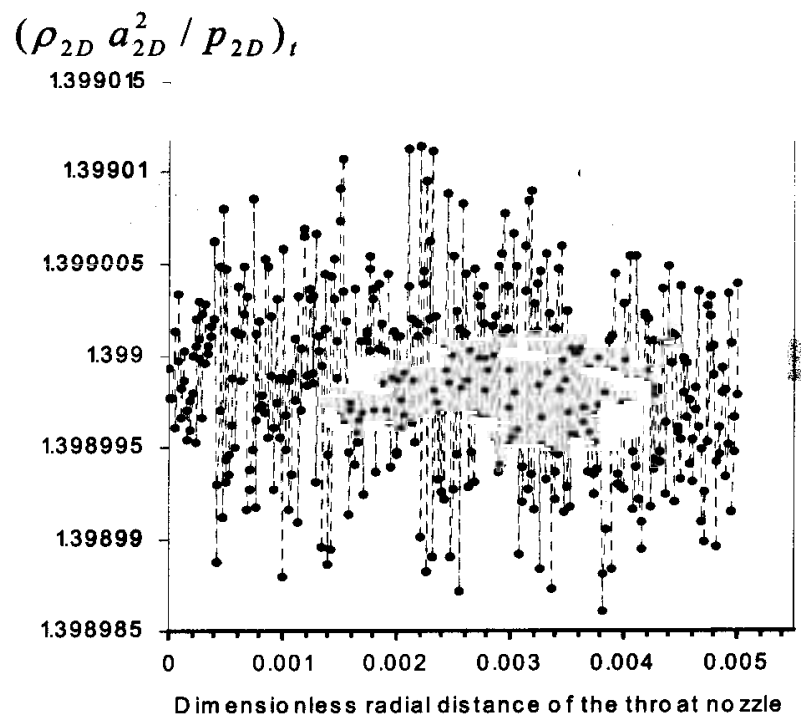

Flgure 8 . Values of the parameter $\left(\rho_{2 D} a_{2 D}^{2} / p_{2 D}\right)_{t}$ in the radial plane of the throat sonic nozzle

The dispersion shown in this graph is very small, of a $1 \times 10^{-6}$ order, and has a tendency toward a constant value of the term $\left(\rho_{2 D} a_{2 D}^{2} / p_{2 D}\right)_{t}$; this factor is determined by means of the numerical integration of these points by the trapezoidal integration method.

To carry out the numerical simulation of the flow, air was used as working fluid; in this case, the value of $y$ is similar to 1.4002389947543, this value is valid for isentropic and ideal flow at a stagnation temperature of $293.15 \mathrm{~K}$. Therefore, the discharge coefficient in the nucleus of the flow can be obtained by equation (18), as follows

$$
C_{d-i n v}=\int_{0}^{1}\left(\frac{\gamma_{0}}{\gamma} \frac{\rho_{2 D}}{\gamma_{o}} \frac{a_{2 D}^{2}}{p_{2 D}}\right) d \bar{Y}=0.9990
$$




\section{RESULTS}

By substituting equations (10) and (18) in equation (6), an analytical correlation to characterize the discharge coefficient is obtained by means of the numerical and theoretical approaches, as shown in equation (19)

$$
C_{d}=0.9990-0.09970 \operatorname{Re}_{d}^{-0.2113564}
$$

In the above expression, the first term, physically represents the discharge coefficient for an inviscid flow (i. e., $R \boldsymbol{e}_{i d e a l}$ $\rightarrow \infty$ ) and it's a measure of the influence of multi-dimensional phenomena on the discharge coefficient. The second term is proportional to the reduction in $C_{d}$ due to viscous effects, I. e., the boundary layer thickness at the sonic nozzle throat. It is important to point out that the obtained model was validated with Chol's experimental correlation of the discharge coefficient obtained at the KRISS [10]. In that study, three sonic nozzles with throat diameters of $4.020,8.079$, and $13.433 \mathrm{~mm}$, nominal volumetric flowrates of 10,36 , and $100 \mathrm{~m}^{3} / \mathrm{h}$, respectively, and stagnation pressure from 1.0 to $2 \mathrm{MPa}$ and throat Reynolds numbers from 1.4 to $2.7 \times 10^{6}$ were calibrated. Besides, in that work the experimental values of the discharge coefficient were expressed by an experimental correlation in function of the Reynolds number of the throat sonic nozzle with an uncertainty of $\pm 0.00068 \%$. This correlation is given by equation (20)

$$
C_{d}=0.99575-3.7026 / \operatorname{Re}_{d}^{0.5}
$$

On the other hand, the obtained correlation was also compared with the $150-9300$ experimental correlation. This correlation is given by equation (21) and is valid in the range of the throat Reynolds numbers of $10^{5}<\operatorname{Re}_{\mathrm{d}}<10^{7}$ with an uncertainty of $\pm 0.5 \%$

$$
C_{d}=0.9935-.525 / \operatorname{Re}_{d}^{0.5}
$$

Figure 9 depicts the deviation between the proposed correlation curves with the curves of the experimental correlations by Choi (KRISS) and ISO-9300, equations (20) and (21), respectively. The results exhibit the same trends (I.e., their slopes are almost identical). From figure 9, it can be observed that the proposed correlation are in good agreement with the experimental correlations by Choi and ISO-9300, the largest difference between the curves is less than $0.2 \%$ in magnitude, this seems to suggest that the proposed correlation characterizes adequately the viscous and multi-dimensional effects present in ISO-9300 nozzles.

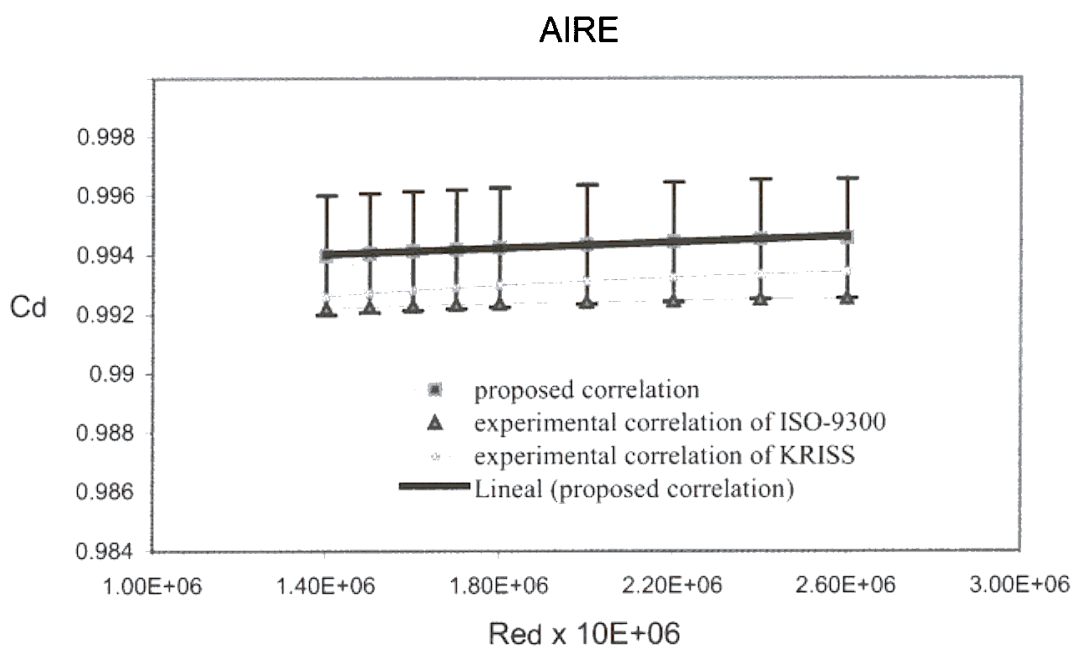

Figure 9. Comparisons between the proposed correlations and experimental correlations of KRISS and ISO-9300 of the discharge coefficient 
From table I, we can easily percelve a tendency for the relative deviation between theoretical and experimental results $\Delta \%$. It can be seen that the accuracy of the present numerical and analytical method for predicting the discharge coefficlent with the experimental correlations of ISO and KRISS can reach a high level: its relative deviation from experimental data $\Delta$ is about $0.2 \%$.

Table I. Comparisons between values of the discharge coefficient of the proposed correlation and values of the experimental correlations of KRISS and ISO-9300

\begin{tabular}{|c|c|c|c|c|c|}
\hline NRe $_{\mathrm{d}} \times 10^{6}$ & $\begin{array}{c}\mathbf{C}_{\mathrm{d}} \\
\text { proposed }\end{array}$ & ISO-9300 $_{\mathrm{d}}$ & $\Delta \%$ & KRISS C $_{\mathrm{d}}$ & $\Delta \%$ \\
\hline 1.4 & 0.99399 & 0.99221 & 0.179 & 0.99262 & 0.138 \\
\hline 1.5 & 0.99406 & 0.99225 & 0.18 & 0.99273 & 0.134 \\
\hline 1.6 & 0.99413 & 0.99229 & 0.185 & 0.99282 & 0.132 \\
\hline 1.7 & 0.99419 & 0.99233 & 0.187 & 0.99291 & 0.129 \\
\hline 1.8 & 0.99425 & 0.99236 & 0.19 & 0.99299 & 0.127 \\
\hline 2.0 & 0.99435 & 0.99242 & 0.194 & 0.99313 & 0.123 \\
\hline 2.2 & 0.99444 & 0.99247 & 0.198 & 0.99325 & 0.12 \\
\hline 2.4 & 0.99453 & 0.99252 & 0.2 & 0.99336 & 0.118 \\
\hline 2.6 & 0.99455 & 0.99255 & 0.2 & 0.99345 & 0.111 \\
\hline
\end{tabular}

\section{CONCLUSIONS}

A numerical procedure for the inviscid flow in a critical venturi nozzle and an improvement over the boundary layer calculation is presented in the paper, aimed at characterizing the discharge coefficient in toroidal throat sonic nozzles ISO-9300. The characterization of this coefficient allows obtaining the parameters that take part in the determination of the discharge coefficient by the analytical and numerical approach. Two main factors that influence directly the discharge coefficient are characterized: boundary layer accumulation along the wall nozzle due to viscous effects that can be calculated by means of boundary layer displacement thickness $\delta$ and the curvature of the sonic line attributed to the multi-dimensional flow field. The combined effect of these non-ideal mechanisms is modeled as a superposition of the individual effects; as a result, each of these non-ideal mechanisms can be analyzed and characterized independently from the influence of the other mechanisms. As a result of this analysis, a characterization of the inviscid discharge coefficient and viscous discharge coefficient was obtained. The connection of these two coefficients yielded a new correlation of the discharge coefficient expressed in function of the Reynolds number of the throat sonic nozzle. The accuracy of the obtained correlation was compared with the correlation of ISO-9300 and the experimental correlation of the discharge coefficient obtained at the KRISS and reported by Y. M. Choi [10], for throat Reynolds numbers from 1.4 to $2.6 \times 10^{6}$. The deviation of the proposed correlation with the proposed correlations by ISO-9300 and KRISS is better than $0.2 \%$. The discharge coefficient considers the no isentropic and non-one-dimensional flow effects existing in the flow of a real gas in the sonic nozzle and relates the flowrate of a real gas to the flowrate for the one-dimensional isentropic steady flow for an ideal gas at critical sonic condition. The thermodynamical behavior of the real gas is characterized by the critical factor flow $C^{*}$, this parameter was determined by johnson [2]. The analytic and numerical characterization of this coefficient allows calibrating directly the nozzle.

The method presented in this work is applicable to flow conditions for which laminar to turbulent transition occurs at short distances upstream the nozzle inlet, taking the boundary layer as totally turbulent.

\section{REFERENCES}

[1] Measurement of fluid flow in closed conduits. Section 1.3 Method of measurement of gas flow by means of critical flow Venturi nozzles, ISO 9300.1990.

[2] Johnson, R. C. Calculations of real gas effects in flow through critical flow nozzles, ASME J. Basic Eng., Series D, 86 , Sept. pp. 519-526, 1964. 
[3] Stratford, B. S. The calculation of the discharge coefficient of profiled choked nozzles and optimum profile for absolute air flow measurement. J. Royal Aeronautic Soclety 68 pp.237-245, 1964.

Tang, S. P. Discharge coefficients for critical flow nozzles and their dependence on Reynolds number. Ph. D. Thesis Dissertation Department of Mechanical Engineering, Princeton Univ., Princeton NJ., 1969.

Geropp, D. Laminaire grenzschichten in ebenen und rotationssymmetrischen Lavalduessen, Deutsche LuftUnd Raumfart, Forschungsbericht, pp. 71-90, 1971.

[6] Ishibashi, M. and Takamoto, M. Very accurate analytical calculation of the discharge coefficients of critical Venturi nozzles with laminar boundary layer, Proceedings of ASME Fluids Engineering Division Summer Meeting, Vancouver, British Columbia, Canada, June, pp. 22-26, 1977.

[71 Johnson, A. N. and Mattingly, G.E. Numerical characterization of the discharge coefficient in critical nozzles, Ph. D. Thesis National Institute Standards and Technology, Gaithersburg, Maryland 20899, technical paper 1989.

[8] Cruz, J. A. and Sanchez, S. F. Parametric Study of Discharge Coefficient on Critical Sonic Nozzles ISO-9300 with Turbulent Boundary Layer, FEDSM-ASME, Montreal, Canada, 2002.

[9] Steger, J. L. Implicit, finite difference simulation of flow about arbitrary geometries, AIAA Journal 16 (7) (1978).

[10] Choi, Y. M., Park, K. A., and Park, J. T. Interference effects of three sonic nozzles of different throat diameters in the same meter tube, Flow Measurement and Instrumentation 10. pp. 175-181, 1991. 